\title{
Edge slip zone size of misaligned common edge contacts
}

\author{
Z. Clark ${ }^{\mathrm{a}}$, R. Ramesh ${ }^{\mathrm{a}, *}$, D.A. Hills ${ }^{\mathrm{a}}$ \\ ${ }^{a}$ Department of Engineering Science, University of Oxford, Parks Road, OX1 3PJ, Oxford, UK.
}

\begin{abstract}
We investigate the effect of a slight misalignment on the behaviour of common edge contacts, developing a procedure for estimating the extent of slip at the edge. Better estimations, compared to one inferred from Williams' solution, are found by introducing the effects of slip and further geometry. The refined asymptote is compared with the exact answer for an example problem.
\end{abstract}

\section{Introduction}

There are four fundamental types of contact: the first is 'incomplete', where the size of the contact increases with applied normal force. The second type is 'complete' where the size of the contact is fixed by one of the bodies and does not change with normal force. The third type is 'receding' where an increase in normal force causes the contact to become smaller. The final type is 'common edge' where the size of the contact is fixed and determined by both bodies. Here we are considering common edge contacts. A full review of contacts can be found in [1].

It is difficult to develop general solutions for common edge contacts because the behaviour is strongly influenced by the overall geometry. Another difficulty is that we are also unable to use half-plane theory as neither body is capable of being idealised as a half plane. It is the only class of contact where the tractions at the edge are neither bounded nor singular, but instead are finite. Despite these difficulties, we are interested in common edge contacts because they are found in many engineering applications. For example, at the big end of a marine diesel connecting rod clamping a balancing weight of the same nominal size as the bearing housing, the interface can develop fretting problems. If we better understood the behaviour of this class of contact, we may be able to design components to maximise life.

Motivated by this, an investigation of two identical cylinders in end on contact was carried out by [2, 3]. The plane form of this problem led to a study of semi-infinite strips in contact under partial slip [4]. Another geometry of interest is a semi-infinite wedge with a line interface, the condition and location of first slip was found [5].

In most types of contact, a relative rigid body translation does not affect the geometry of the problem and hence its solution. Their relative positions before they are brought into contact do not matter. This is not the case for common edge contacts, as any misalignment can lead to vastly different behaviour. In this paper we investigate the effect of a misalignment on possible slip.

\section{Formulation}

An example geometry is given in Figure 1a where the origin is chosen as the midpoint of the contact region. We will initially assume that the coefficient of friction, both for the prototype \& the geometrically simpler model soon to be introduced, is sufficiently high to inhibit all slip. To study the behaviour local to the edge, we can ignore any remote

\footnotetext{
${ }^{*}$ Corresponding author: rangarajan.ramesh@eng.ox.ac.uk
} 
features and consider only the edge of the interface as shown in Figure 1b. The problem has reflectional symmetry so the behaviour at either edge is identical. At any free surface, the only stress allowed is parallel to the free surface so we hypothesise that if $d<<a$ in the example problem Figure 1a, the uniform direct stress, $\sigma_{0}$, alone dominates the near edge behaviour. The accuracy of this assumption will become clear when the example problem is considered. If we zoom in even further, the problem looks identical to a sharp notch as in Figure 1c. At this scale, provided that the coefficient of friction inhibits all slip, we can use the Williams' solution [6, 7] to understand the behaviour at the interface.

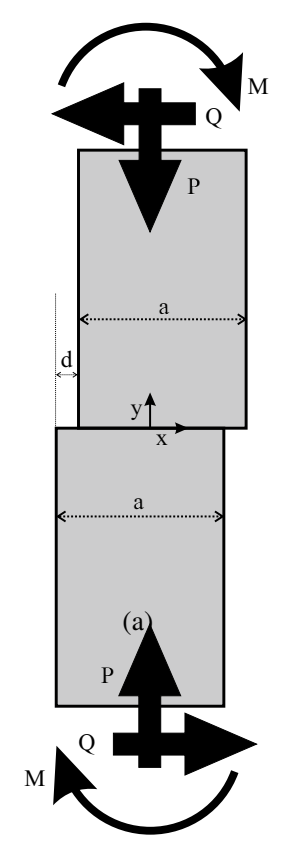

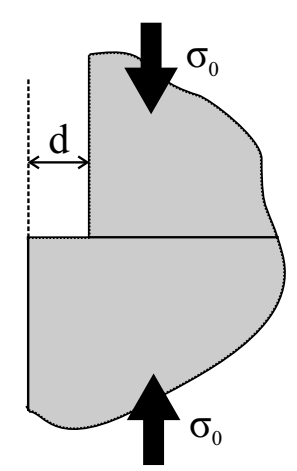

(b)

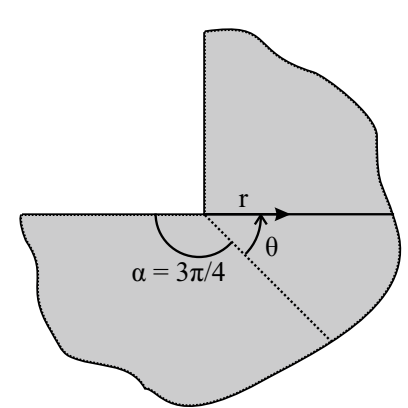

(c)

Figure 1: (a) Example slightly misaligned contact problem; (b) Misaligned edge; (c) A sharp notch

\subsection{Williams' solution}

The state of stress in a semi-finite, sharp notch may be written as a series representation developed by Williams' [6]. Assuming that all of the loading is in-plane, the two singular terms, which dominate the behaviour close to the root, are

$$
\sigma_{i \theta}(r, \pi / 4)=K_{I} r^{\lambda_{I}-1} f_{i \theta}^{I}(r, \pi / 4)+K_{I I} r^{\lambda_{I I}-1} f_{i \theta}^{I I}(r, \pi / 4) \quad i=r, \theta
$$

When $2 \alpha=3 \pi / 2$, the eigenvalues are, $\lambda_{I}=0.5445$ and $\lambda_{I I}=0.9085$. The functions $f_{i j}^{I}(r, \theta)$ and $f_{i j}^{I I}(r, \theta)$ are given in Appendix A.

Because $\lambda_{I}<\lambda_{I I}$, very close to a notch root, the mode $I$ term will dominate the solution. This means that as a first crude approximation of the behaviour, we can consider just the first term from Equation 1. We can draw on results derived for the edges of complete contacts [8] which employ the eigenvectors. For the geometry we are considering $(2 \alpha=3 \pi / 2)$, the traction ratio along the adjacent contact interface is given by

$$
-\frac{\left|\sigma_{r \theta}\right|}{\sigma_{\theta \theta}}=-\frac{f_{r \theta}^{I}(\pi / 4)}{f_{\theta \theta}^{I}(\pi / 4)}=0.5431 .
$$

This result is given by the black horizontal line in Figure 2.

If the contact edge is perfectly aligned a very small coefficient of friction is sufficient to ensure edge stick. However, if there is a step present at the interface and $f<0.543$ the edge of the contact will experience slip. If it is greater than 
this value, the contact will remain adhered and under changing remote loads the edge will be the last point to slip. These results are based solely on the dominant term in the Williams' solution. We can improve the domain of validity of the approach by incorporating both singular terms, i.e. matching the wedge shown in Figure 1c to the semi-infinite step in Figure 1b. A Finite Element (FE) model was developed for the geometry shown in Figure 1b, with the interface bonded, and the stress intensity factors found (the process is described in [9].) They are

$$
\left[\begin{array}{l}
K_{I} d^{1-\lambda_{I}} \\
K_{I I} d^{1-\lambda_{I I}}
\end{array}\right]=\left[\begin{array}{l}
-0.5764 \\
-0.5842
\end{array}\right] \sigma_{0} .
$$

The traction ratio close to the edge for the two term solution is given by the blue dashed line in Figure 2 along the contact interface. The figure also includes the traction ratio inferred directly from the FE solution (red chain dashed line), so we have a one-term, two-term and within the limitation of numerical solution, an 'exact' answer for the geometry shown in Figure 1b. It can be seen that the two term solution gives a good approximation when $r / d<1$. This self-contained solution has yet to be fitted into the finite problem, Figure 1a and by implication, is independent of the loading details of the example. The extent of slip may be estimated by finding the value of $r / d$ where the traction ratio exceeds the coefficient of friction. The two magenta lines which consider the finite problem in Figure 1a will be discussed in section 2.2.

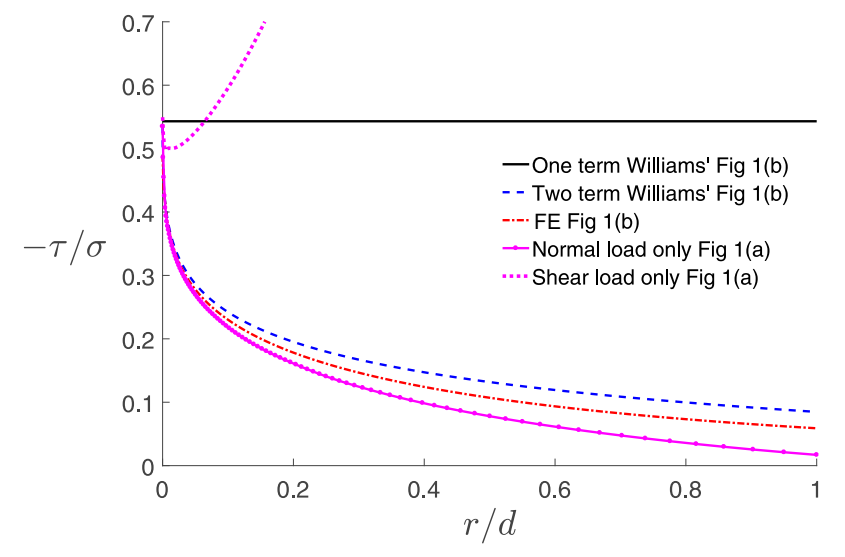

Figure 2: Estimation of the traction ratio near the edge $(f>0.5431)$

\subsection{Introduction of slip}

A new FE contact model of Figure $1 \mathrm{~b}$ was made in which the loading is maintained but frictional slip is permitted. The radius of the overall model is $100 d$ to approximate two infinite half planes. A plane strain, structured mesh is employed with a minimum size of $0.001 \mathrm{~d}$ at the edge of the contact. The Lagrange Multiplier friction formulation is used. The extent of slip at the edge of the contact is shown in Figure 3 by the solid black line. The figure also includes the implied (from violations) slip zone size deduced form the monolithic model, but with violation of the friction law (red chain dotted line in Figure 2 implies red chain dotted line in Figure 3) The shear and normal tractions are excited by the same load hence the result is independent of the magnitude of loading. The bilateral (monolithic) solution significantly under-estimates the extent of slip.

Figure 4 compares the slip zone size from Figure $1 \mathrm{~b}$ with that of the finite problem in Figure 1a. It can be seen that at a low ratio of $Q / f P$ the prediction is good but it significantly worsens at higher ratios. To understand this, we show in Figure 2 the traction ratio in the finite problem for normal only and shear only loading. The traction ratio due to the normal load, 


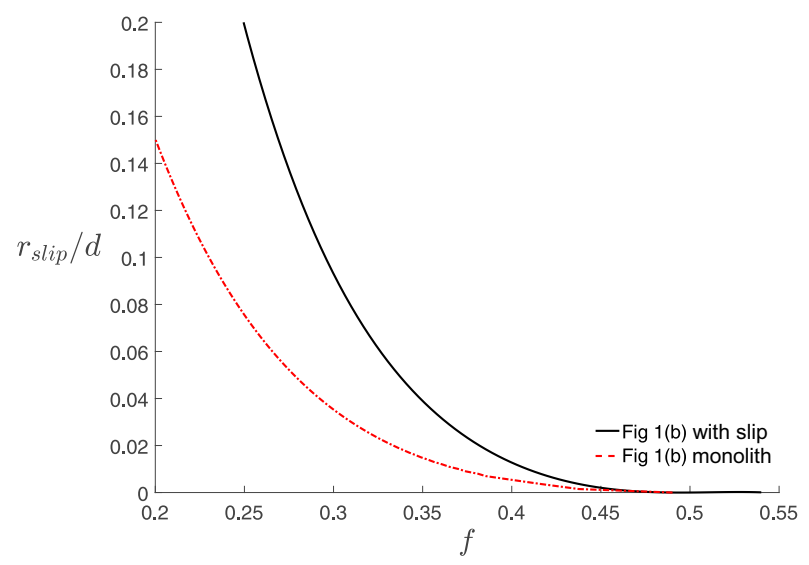

Figure 3: Effect of slip

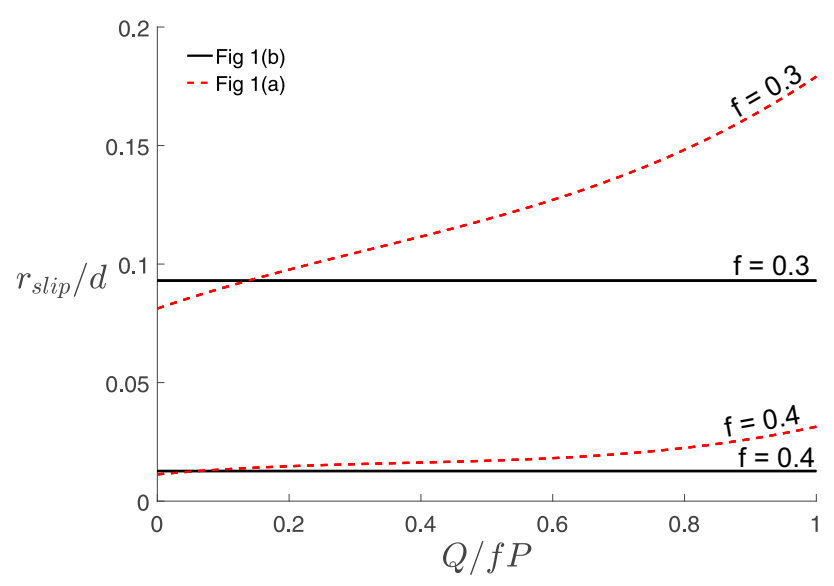

Figure 4: Effect of shear loading

is approximated well over the region of concern however, the traction ratio for a shear load is of a very different form and poorly approximated. This explains why the inclusion of a shear load worsens the estimate of the slip zone in the finite problem.

\section{Example problem: Further considerations}

\subsection{Fully aligned contact}

Figure 1a shows an example problem, in which two beams of equal width, $a$, and length $5 a$, are pressed together by a normal force and then subject to a shear force and moments. The loads are such that the internal moment falls to zero at the interface whilst ensuring that there is no rigid body rotation from the shear force. For the case with no misalignment $(d / a=0)$ the tractions $(\sigma, \tau)$ may be written down explicitly

$$
\sigma(x)=\frac{P}{2 a} ; \quad \tau(x)=\frac{3 Q}{4 a}\left(1-\frac{x^{2}}{a^{2}}\right) .
$$

It is obvious that the first place to slip will be at the centre of the contact, and the last place will be the edge. It is also obvious that the condition for first slip is given by $Q / f P=2 / 3$. 


\subsection{Effect of misalignment}

In the presence of a misalignment $(d / a>0)$, the value of the coefficient of friction will determine the behaviour of the contact. The problem is coupled, that is to say, the normal load gives rise to both a normal and shear tractions, and similarly for the shear force. The behaviour we can expect is given in Figure 5 for a misalignment of $d / a=0.1$.

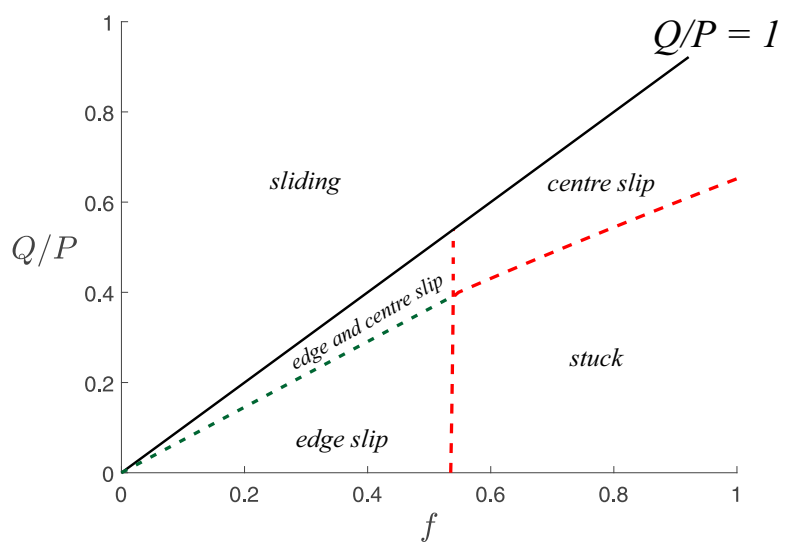

Figure 5: Behaviour with different loads and friction $(d / a=0.1)$

If $f<0.5431$, slip is present at the edge regardless of $Q / P$. Increasing the value of $Q / P$ causes the slip zone to grow from the edge as well as nucleating from the centre, until the beams slide. The red dashed line is found from the FE monolith model by looking at the contribution to the tractions, $p(x)$ and $q(x)$ from $P$ and $Q$ individually and determining the coefficient of friction required to maintain full stick for any combination. The green dashed line demarcating the onset of centre slip in Figure 5 is approximate. If $f>0.5431$, there is no slip initially under a normal load alone, and only begins, from the centre of the contact, when $Q / P$ reaches the red dashed line in Figure 5. Increasing the shear force means that slip starts at the centre and grows towards the edges until sliding. It is interesting to note that a small misalignment of the contact drastically changes the behaviour both at the edges and at interior points. Figure 5 tells us about the presence of slip, but not its extent.

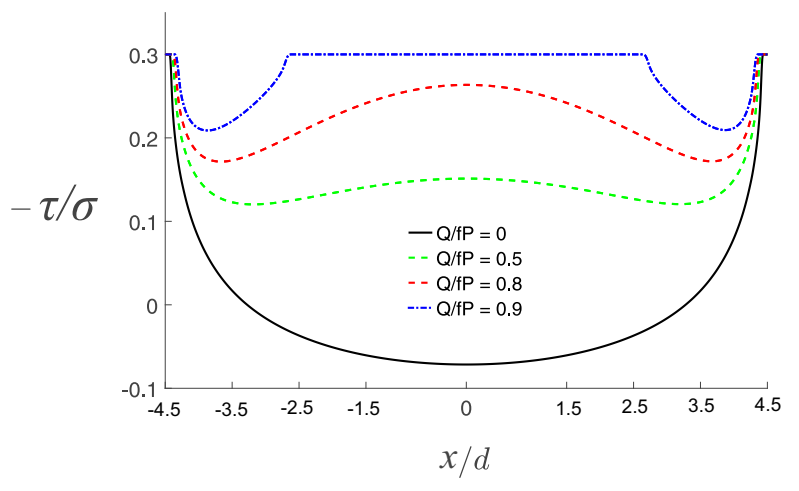

Figure 6: Traction ratio across the whole contact $(f=0.3)$

To look at the extent of slip, we continue to look at an example with a misalignment $d / a=0.1$ which is probably the largest offset where an asymptote is of value, and construct a FE model including a frictional interface. The tractions across the contact can be seen in Figure 6 for, $f=0.3$. It can be seen that the traction ratios for different values of $Q / f P$, although vastly different internally, are similar at the edge. An interesting effect of the misalignment is the higher values of $Q / f P$ required to cause centre slip. At $Q / f P=0.8$ there is no interior slip which wouldn't be the case for the aligned contact. Furthermore, when the shear loading is increased such that $Q / f P=0.9$ there is a large region of central slip. 
At this coefficient of friction we estimate, from Figure 4 , a slip intrusion from the edge $r_{\text {slip }} / d \approx 0.09$. The actual slip extent, too small to show on Figure 6 is given by the red dotted line in Figure 4.

\section{Conclusion}

A procedure to estimate the size of the slip zone in misaligned common edge contacts is presented. We find that there is always slip at the edge if the coefficient of friction, $f<0.543$. Above this coefficient of friction, the edge is the last point to slip. The extent of this slip for low values of $Q / f P$ is accurately estimated by incorporating the effect of slip in a semi-infinite model, that only considers one edge of the contact, with normal loading alone.

It is surprising how quickly the 'monolith with slip violation' solution diverges from the true solution in Figure 3, but the coefficients of friction where this happens are unrealistically small. The procedure gives a reasonable estimate of the slip extent for coefficients of friction just below that to achieve stick, and these are those those likely to be practically encountered. The Williams' solution captures the stepped edge behaviour well when the remote load is a uniform direct stress.

\section{References}

[1] R. Ramesh and D. A. Hills. Recent progress in understanding the properties of elastic contacts. Proceedings of the Institution of Mechanical Engineers, Part C: Journal of Mechanical Engineering Science, 229(12):2117-2126, 2015.

[2] R. Ramesh and D. A. Hills. The condition for first slip in a common edge contact subject to in-plane and anti-plane loading. The Journal of Strain Analysis for Engineering Design, 50(6):386-390, 2015.

[3] M.E. Kartal, D.A. Hills, D. Nowell, and J.R. Barber. Torsional contact between elastically similar flat-ended cylinders. International Journal of Solids and Structures, 47(10):1375 - 1380, 2010.

[4] M.E. Kartal, J.R. Barber, D.A. Hills, and D. Nowell. Partial slip problem for two semi-infinite strips in contact. International Journal of Engineering Science, 49(2):203 - 211, 2011.

[5] D.A. Hills and D. Dini. Common edge contacts: Effect of interface line orientation. International Journal of Mechanical Sciences, 81:73 - 76, 2014.

[6] M.L. Williams. Stress singularities resulting from various boundary conditions in angular corners of plates in extension. J. Appl Mech., 19:526-528, 1952.

[7] J. R. Barber. Elasticity. Dordrecht, 3rd Ed., 2010.

[8] D. A. Hills and D. Dini. What level of friction guarantees adhesion in a complete contact? The Journal of Strain Analysis for Engineering Design, 39(5):549-551, 2004.

[9] R. C. Flicek. Analysis of complete contacts subject to fatigue. PhD thesis, University of Oxford, 2015.

\section{Appendix A. Williams' eigenvectors}

$$
\begin{gathered}
f_{\theta \theta}^{I}(r, \theta)=\frac{\cos \left[\left(\lambda_{I}-1\right) \alpha\right] \cos \left[\left(\lambda_{I}+1\right) \theta\right]-\cos \left[\left(\lambda_{I}+1\right) \alpha\right] \cos \left[\left(\lambda_{I}-1\right) \theta\right]}{\cos \left[\left(\lambda_{I}-1\right) \alpha\right]-\cos \left[\left(\lambda_{I}+1\right) \alpha\right]} \\
f_{r \theta}^{I}(r, \theta)=\frac{\sin \left[\left(\lambda_{I}-1\right) \alpha\right] \sin \left[\left(\lambda_{I}+1\right) \theta\right]-\sin \left[\left(\lambda_{I}+1\right) \alpha\right] \sin \left[\left(\lambda_{I}-1\right) \theta\right]}{\sin \left[\left(\lambda_{I}-1\right) \alpha\right]-\frac{\left(\lambda_{I}+1\right)}{\left(\lambda_{I}-1\right)} \sin \left[\left(\lambda_{I}+1\right) \alpha\right]} \\
f_{\theta \theta}^{I I}(r, \theta)=\frac{\sin \left[\left(\lambda_{I I}-1\right) \alpha\right] \sin \left[\left(\lambda_{I I}+1\right) \theta\right]-\sin \left[\left(\lambda_{I I}+1\right) \alpha\right] \sin \left[\left(\lambda_{I I}-1\right) \theta\right]}{-\sin \left[\left(\lambda_{I I}-1\right) \alpha\right]+\frac{\left(\lambda_{I I}-1\right)}{\left(\lambda_{I I}+1\right)} \sin \left[\left(\lambda_{I I}+1\right) \alpha\right]} \\
f_{r \theta}^{I I}(r, \theta)=\frac{\cos \left[\left(\lambda_{I I}-1\right) \alpha\right] \cos \left[\left(\lambda_{I I}+1\right) \theta\right]-\cos \left[\left(\lambda_{I I}+1\right) \alpha\right] \cos \left[\left(\lambda_{I I}-1\right) \theta\right]}{\cos \left[\left(\lambda_{I I}-1\right) \alpha\right]-\cos \left[\left(\lambda_{I I}+1\right) \alpha\right]}
\end{gathered}
$$

